\title{
SOME C*-ALGEBRAS ASSOCIATED TO QUANTUM GAUGE THEORIES
}

\author{
KEITH C. HANNABUSS
}

(Received 30 July 2010; accepted 24 February 2011)

Communicated by S. Paycha

Dedicated to Alan Carey, on the occasion of his 60th birthday

\begin{abstract}
Algebras associated with quantum electrodynamics and other gauge theories share some mathematical features with $T$-duality. Exploiting this different perspective and some category theory, the full algebra of fermions and bosons can be regarded as a braided Clifford algebra over a braided commutative boson algebra, sharing much of the structure of ordinary Clifford algebras.
\end{abstract}

2010 Mathematics subject classification: primary 81T13; secondary 81T75, 46L08, $18 \mathrm{D} 10$.

Keywords and phrases: quantum electrodynamics, gauge theories, noncommutative geometry, Rieffel inducing, monoidal dagger categories, bar categories, braiding.

\section{Introduction}

It is just over 60 years since quantum electrodynamics achieved its modern form [14, 25, 26]. Some insights into its ultraviolet and infrared divergences have been provided by causal distribution splitting [15, 16, 24], and the Hopf algebra structure of nested Feynman diagrams [12, 19], respectively, both of which fit naturally within noncommutative geometry [17, Ch. 12-13]. With that in mind, we present a slightly different perspective on the operator algebras of gauge theories, which emphasizes noncommutative geometric features, and also parallels some techniques which appear in string theory and $T$-duality [5, 20].

For abelian gauge theories it is possible to give an explicit derivation of their algebraic structures in terms of inducing and crossed products. This parallels other examples in noncommutative geometry, and the inducing process gives rise to the Poisson-Gauss law relating the gauge field and fermion charge density. These ideas are sketched in the next four sections. 
The remainder of the paper elucidates some features of the algebraic construction more generally, using its functorial properties. This reveals that the fermionic part of the theory can be considered as a braided Clifford algebra over the gauge bosonic algebra.

\section{Fermions}

The conventional operator approach to quantum electrodynamics (QED) combines a fermionic anticommutation relation algebra (CAR) and a bosonic commutation relation algebra (CCR). We shall start with the fermions and introduce the bosons via a gauge principle. For convenience, we work a Hamiltonian rather than a Lagrangian approach, so that we work over space $X=\mathbb{R}^{3}$ rather than over space-time, and in a radiation gauge.

The real structure of the anticommutation relations, encoded in a complexified Clifford algebra $\operatorname{Cliff}(W, Q)$ of a real pre-Hilbert space $W$ with inner product $Q$, turns out to be more fundamental. The Clifford algebra is the unital algebra generated by elements $\Psi(\xi)$, so that for any cross-sections $\xi, \eta \in W$, the (equal time) anticommutator satisfies

$$
[\Psi(\xi), \Psi(\eta)]_{+}:=\Psi(\xi) \Psi(\eta)+\Psi(\eta) \Psi(\xi)=2 Q(\xi, \eta) 1 .
$$

This algebra has a natural antilinear antimorphism or *-structure, and a normalized trace $\tau: \operatorname{Cliff}(W, Q) \rightarrow \mathbb{C}$ [21]. In QED one can take $W$ to be the smooth, rapidly decreasing (more precisely, Schwartz) cross-sections of a Dirac spinor bundle over $\mathbb{R}^{3}$, considered as a real vector space, and $Q(\xi, \eta)$ the integral over $\mathbb{R}^{3}$ of the real part of the spinor inner product $\langle\xi(\mathbf{x}), \eta(\mathbf{x})\rangle$.

For any complex structure $J$ on $W$, we can form the complex *-algebra generated by elements $\Psi_{J}(\xi)=\frac{1}{2}(\Psi(\xi)-i \Psi(J \xi))$, which satisfy the anticommutation relations

$$
\left[\Psi_{J}(\xi)^{*}, \Psi_{J}(\eta)\right]_{+}=\langle\xi, \eta\rangle=\int_{\mathbb{R}^{3}}\langle\xi(\mathbf{x}), \eta(\mathbf{x})\rangle d^{3} \mathbf{x}
$$

(with $\Psi_{J}$ a creation operator). The procedure can be reversed by taking selfadjoint operators $\Psi(\xi)=\Psi_{J}(\xi)+\Psi_{J}(\xi)^{*}$ which satisfy the original Clifford algebra relations. In the CAR, $\xi$ and $\eta$ may be regarded as elements of the complexification $W_{\mathbb{C}}=W \otimes \mathbb{C}$. This is the direct sum of two complex subspaces $W_{J}^{ \pm}=\operatorname{ker}(1 \pm i J)$, each of which is easily checked to be isotropic with respect to the complex bilinear extension of $Q$. Formally,

$$
\Psi_{J}(\xi)=\frac{1}{2}(\Psi(\xi)-i \Psi(J \xi)) \sim \Psi\left(\frac{1}{2}(1-i J) \xi\right)
$$

so that we may as well take $\xi \in W_{J}^{+}$. Similarly, in $\Psi_{J}(\eta)$ we can take $\eta \in W_{J}^{-}$.

We can now define the Fock representation of the CAR associated with $J$ which takes place on the Hilbert space completion of the exterior algebra $\wedge W_{J}^{+}$equipped with the inner product derived by extending $Q$. The creation operator $\Psi_{J}(\xi)$ acts as 
exterior multiplication by $\xi \in W_{J}^{+}$. Its adjoint turns out to be an inner multiplication and the CAR can easily be verified [21]. This Fock representation has an obvious cyclic vector $\Phi_{J}=1 \in \mathbb{C}=\bigwedge^{0} W_{J}^{+}$, called the Fock vacuum, which is annihilated by all the $\Psi_{J}(\xi)^{*}$. This provides a correspondence between complex structures $J$ and vacuum states $\Phi_{J}$. Different physical states correspond to different complex structures and different complex structures usually give inequivalent Fock representations.

\section{The gauge bosons}

Quantum electrodynamics was soon followed by nonabelian gauge theories, and now gauge symmetries are regarded as fundamental. This insight provides an alternative way to the traditional introduction of gauge bosons simply by adding new generators and commutation relations. Let $G$ be the (global) gauge group (U(1) for QED, and generally a compact connected Lie group), which we suppose to have a unitary representation on spinors. The natural pointwise action of the local gauge group of smooth maps tending to 1 at $\infty, \mathcal{G}=\operatorname{Map}_{0}\left(\mathbb{R}^{3}, G\right)$, on the space $W$ of sections of the spin bundle, preserves the inner product $Q$, and for $\chi \in \mathcal{G}$ we may define an automorphism $\alpha_{\chi}$ of $\operatorname{Cliff}(W, Q)$ by $\alpha_{\chi}[\Psi(\xi)]=\Psi(\chi \cdot \xi)$. (In QED one has $G=\mathrm{U}(1)$ which certainly has a unitary representation on the complex spinors.) When $G=\mathrm{U}(1)$ the abelian gauge group, $\mathcal{G}$, is amenable; Carey and Grundling showed that when $G=\mathrm{U}(n)$ or $\mathrm{SU}(n)$, and also for smooth subalgebras on compactified $\mathbb{R}^{3}$, there is still a topology with respect to which $\mathcal{G}$ is amenable [9].

This action of the gauge group is not compatible with the Dirac equation governing the dynamics of the fermions, so we introduce a connection $\nabla$ on the spinor bundle, which compensates for the fermionic gauge transformation by changing to $\chi^{-1} \nabla \chi$ under the gauge group action. In practice it is easier to replace $\nabla$ by its Lie algebra $\mathfrak{g}$ valued connection 1-form $\omega \in \Omega=\Omega^{1}(X, \mathfrak{g})$, and then the gauge group action is given by $\omega \mapsto \chi^{-1} \omega \chi+i \chi^{-1} d \chi$. For an abelian group $G$ such as U(1) this simplifies to $\omega \mapsto \omega+i \chi^{-1} d \chi$.

The fermion dynamics depend on $\omega$, and for each connection $\omega$ there is a fermion space $W_{\omega}$, giving a pre-Hilbert bundle $W$ over the space of connections, and an associated Clifford bundle with fibres $\operatorname{Cliff}\left(W_{\omega}, Q\right)$. So fermions should now be regarded as cross-sections of this bundle. The gauge principle requires that the change of connection agrees with the automorphism $\alpha_{\chi}$. That is, for a Clifford algebra valued section $\Xi$ of the bundle over connections one has

$$
\Xi\left(\omega+i \chi^{-1} d \chi\right)=\alpha_{\chi}[\Xi(\omega)]
$$

precisely the condition defining an induced algebra:

$$
\operatorname{ind}_{\mathcal{G}}^{\Omega}(\text { Cliff, } \alpha)=\left\{\Xi \in \text { Cliff : } \Xi\left(\omega+i \chi^{-1} d \chi\right)=\alpha_{\chi}[\Xi(\omega)]\right\}
$$

Similar algebras appear in string theory, where the infinite-dimensional vector group $\Omega$ is replaced by a locally compact vector group, and the gauge group $\mathcal{G}$ by a 
maximal rank lattice subgroup, so that the quotient $\Omega / \mathcal{G}$ is a torus, and more generally one studies principal torus bundles, represented by continuous trace algebras with nontrivial Dixmier-Douady class (or $H$-flux) [5, 20]. Nontrivial classes seem to be unnecessary for our abelian gauge theories, though we can still associate the algebra with an $\Omega / \mathcal{G}$-bundle $[10,11]$.

The induced algebra carries a Mackey action of functions on $\Omega / \mathcal{G}$ : any function $F$ on $\Omega / \mathcal{G}$ lifts to $\widetilde{F}$ on $\Omega$ which has a multiplication action on the induced algebra,

$$
(F \cdot \Xi)(\omega)=\widetilde{F}(\omega) \Xi(\omega) .
$$

In our Hamiltonian picture the connection form $\omega$ represents the magnetic vector potential $\mathbf{A}$, and we can identify the multiplication action of $F$ with the action of $\widetilde{F}(e \mathbf{A} / \hbar)$, where $\mathbf{A}$ is the quantized magnetic potential. By construction this depends only on the gauge equivalence class of $\mathbf{A}$.

Since $\Omega$ is not locally compact it is not obvious which topology or algebra of functions to use. Following the usual conventions of algebraic quantum field theory, we start with the $C^{*}$-algebra generated by elements $\phi_{a}$ of a dual group; more precisely, these can be obtained from elements $a$ of a dual vector space $\widehat{\Omega}$ of continuous linear functionals $\Omega$, so that

$$
\phi_{a}(\omega)=e^{i a(\omega)} .
$$

We thus take finite linear combinations of these functions with pointwise multiplication. For the sections of the bundle over the connection space we can similarly use linear combinations of products of spinors with functions $\phi_{a}$.

An infinite-dimensional vector group $\Omega$ lacks a canonical Pontryagin dual. Many spaces are in duality to $\Omega$ (for locally convex spaces the Mackey-Arens theorem characterizes the dual pairs). Besides an algebraic dual, one might take the geometric holonomy dual of the Rovelli-Smolin transform [2, Ch. 14], which takes dual elements labelled by loops $\gamma$ in $\mathbb{R}^{3}$ paired with potentials by the holonomy of the loop

$$
\gamma: \omega \rightarrow \int_{\gamma} \omega .
$$

Whichever dual one uses, the functions lifted from $\Omega / \mathcal{G}$ are given by $a \in \mathcal{G}^{\perp}$, that is, dual elements which vanish on gauge-trivial connections. This shows explicitly that a generalized fixed point algebra exists. (Ashtekar and Lewandowski [3] have shown that the spectrum of the associated unital $C^{*}$-algebra is a compactification of $\Omega / \mathcal{G}$. This could potentially provide another algebra of continuous functions.)

\section{Electric fields}

Dynamically, magnetic fields oscillate into electric fields, so the above description is still incomplete. In string theory one forms the dual crossed product algebra coming from a natural action of $\Omega$, and then $T$-duality turns out to be Takai duality, [30]. We do not need the duality here, but for abelian $G$ we can similarly form a crossed product. 
There is an $\Omega$-action on the induced algebra given by

$$
\tau_{u}[\Xi](\omega)=\Xi(\omega+u)
$$

which one may check to be consistent with the gauge condition. This action allows us to form the crossed product algebra

$$
\mathcal{A}=\operatorname{ind}_{\mathcal{G}}^{\Omega}(\operatorname{Cliff}(W, Q), \alpha) \rtimes_{\tau} \Omega .
$$

We note that, by definition, $\alpha_{\chi}=\tau_{i \chi^{-1} d \chi}$.

The crossed product is effectively generated by the original algebra and the group, considered as point measures $\delta_{u}$ concentrated at $u \in \Omega$, with the covariance property that $\tau_{u}$ is implemented by the adjoint (conjugation) action of $\delta_{u}$. So overall we take the $*$-algebra generated by $\phi_{a, u}=\phi_{a} \delta_{u}$ with product

$$
\phi_{a, u} * \phi_{b, v}=\left(\phi_{a} \delta_{u}\right) *\left(\phi_{b} \delta_{v}\right)=\phi_{a}\left(e^{i b(u)} \phi_{b}\right) \delta_{u} * \delta_{v}=e^{i b(u)} \phi_{a+b} \delta_{u+v} .
$$

This is clearly noncommutative since

$$
\left(\phi_{a, u} * \phi_{b, v}\right)=e^{i b(u)} \phi_{a+b, u+v}=e^{i[b(u)-a(v)]} \phi_{b, v} * \phi_{a, u} .
$$

The $*$ operation is

$$
\phi_{a, u}^{*}=e^{i a(u)} \phi_{-a,-u}
$$

so that the generators are unitary:

$$
\left(\phi_{a, u}^{*} * \phi_{a, u}\right)(\omega, \epsilon)=e^{i a(u)} \phi_{-a,-u} * \phi_{a, u}=e^{i a(u)} e^{-i a(u)} \phi_{0,0}=1 .
$$

In general, the crossed product consists of complex-valued 'functions' on $\Omega \times \Omega$ with product and star

$$
(f * g)(\omega, \epsilon)=\int f\left(\omega, \epsilon_{1}\right) g\left(\omega+\epsilon_{1}, \epsilon-\epsilon_{1}\right) d \epsilon_{1}, \quad f^{*}(\omega, \epsilon)=\overline{f(\omega-\epsilon,-\epsilon)} .
$$

The $\mathcal{G}$-fixed algebra $\mathcal{B}$ can be considered as the algebra generated by $\phi_{a, u}$ with $a \in \mathcal{G}^{\perp}$, the subgroup of dual elements which map $\mathcal{G}$ to 1 . (This demonstrates the existence of the generalized fixed point algebra, which is not always obvious.) Although it is customary to handle the boson algebra in this way, it does yield an algebra with some nonphysical representations. By endowing the groups with a more subtle topology and using continuous functions it is possible to get only the physical, regular, representation, [18].

There is an action of $\Omega$ on the algebra of cross-sections of the Clifford bundle over $\Omega$, and so here too we can form a crossed product algebra, which will described both the fermion and boson fields. The Clifford bundle is graded and its 0-component gives rise to the gauge boson algebra just described,

$$
\mathcal{B}=\operatorname{ind}_{\mathcal{G}}^{\Omega}(\mathbb{C}, \alpha) \rtimes_{\tau} \Omega
$$


(A similar construction occurs in the noncommutative geometric approach to $T$-duality in [20] but with $\Omega$ a finite-dimensional vector group and $\mathcal{G}$ a maximal rank lattice in $\Omega$ so that $\Omega / \mathcal{G}$ is a torus. Then $\operatorname{ind}_{\mathcal{G}}^{\Omega}(\mathbb{C}, \alpha)$ is the $\mathrm{C}^{*}$-algebra representing a principal $\Omega / \mathcal{G}$-bundle, and $\operatorname{ind}_{\mathcal{G}}^{\Omega}(\mathbb{C}, \alpha) \rtimes_{\tau} \Omega$ is the $\mathrm{C}^{*}$-algebra associated with its $T$-dual torus bundle. From this viewpoint $T$-duality is just Takai duality for particular $\mathrm{C}^{*}$-algebras.) In the context of this paper the crossed product is equivalent to the CCR algebra for the bosonic gauge theory, including longitudinal modes. The full Clifford algebra is a module for this boson algebra (though not of finite rank), so that we can consider this as a kind of vector bundle over the noncommutative space associated with the boson algebra. The noncommutativity of the bosons means that an uncertainty principle constrains the fermionic cross-sections.

In QED the canonical commutation relations for smeared gauge boson fields, $\mathbf{A}(\mathbf{a})$, $\mathbf{E}(\mathbf{u}),\left(\mathbf{a}, \mathbf{u}\right.$ in the Schwartz space $\mathcal{S}(X, \mathfrak{g})$, and $\left.\mathbf{E}(\mathbf{u})=\int \mathbf{E}(\mathbf{x}) \cdot \mathbf{u}(\mathbf{x}) d^{3} \mathbf{x}\right)$ are

$$
[\mathbf{E}(\mathbf{u}), \mathbf{A}(\mathbf{a})]=-i \frac{\hbar}{\epsilon_{0}} \int_{\mathbb{R}^{3}} \mathbf{u}(\mathbf{x}) \cdot \mathbf{a}^{\perp}(\mathbf{x}) d^{3} \mathbf{x},
$$

where $\mathbf{a}^{\perp}$ denotes the transverse part. (Henceforth we shall assume that the gauge has been fixed so that $\mathbf{a}$ is transverse, and drop the $\perp$.) This means that the electric field generates translations. More precisely, exponentiating this to the group yields

$$
e^{i \mathbf{u} \cdot \mathbf{E}}\left(\frac{e}{\hbar} \mathbf{A}(\mathbf{a})\right) e^{-i \mathbf{u} \cdot \mathbf{E}}=\left(\frac{e}{\hbar} \mathbf{A}(\mathbf{a})+\frac{e}{\epsilon_{0}} \int \mathbf{a} \cdot \mathbf{u}\right),
$$

so that $\tau_{u}$ is implemented by

$$
\left[\exp \left(\frac{i \epsilon_{0}}{\hbar} \mathbf{E}(\mathbf{u})\right)\right]
$$

where $\mathbf{E}$ is the quantized electric field.

Our Hamiltonian gauge-fixed description is not manifestly Lorentz invariant, since Lorentz transformations mix the magnetic and electric fields (forcing further gauge transformations [6]). This suggests the interesting question of how the Lorentz symmetry manifests itself in the induced crossed product.

\section{The Poisson-Gauss law}

Gauge invariance allows us to remove the longitudinal magnetic fields, but longitudinal electric fields remain as part of the translation group.

For $f \in C_{0}^{\infty}(X)$ set $\chi_{f}=\exp (-i f)$, so that $\chi_{f}^{-1} d \chi_{f}=-i d f$,

$$
\tau_{d f}[\xi](\omega)=\xi(\omega+d f)=\xi\left(\omega+i \chi_{f}^{-1} d \chi_{f}\right)=\chi_{f} \cdot \xi(\omega)=e^{-i f} \xi(\omega) .
$$

By definition, $\tau_{e d f / \epsilon_{0}}$ is implemented in the crossed product by $\exp (i \mathbf{E} \cdot(\nabla f))$, so that implements multiplication by $\exp \left(-i e f / \epsilon_{0}\right)$. 
On the other hand a formal calculation shows the implementor should be $\exp \left(-i \rho(f) / \epsilon_{0}\right)$, where the charge density operator

$$
\rho(f)=\int_{X} f(\mathbf{x}) e \Psi_{J}(x)^{*} \Psi_{J}(x) d^{3} \mathbf{x} .
$$

(We need to work with the complex algebra here to incorporate complex gauge factors.) So, differentiating and using the divergence theorem, we get the PoissonGauss law:

$$
(\nabla \cdot \mathbf{E})(f)=-\mathbf{E} \cdot(\nabla f)=\frac{1}{\epsilon_{0}} \rho(f) .
$$

More directly, we have the differentiated version

$$
\operatorname{ad}((\nabla f) \cdot \mathbf{E})\left[\Psi_{J}(\xi)\right]=\frac{e}{\epsilon_{0}} \Psi_{J}(f \xi) .
$$

Using $\mathbf{u}=\nabla f$, or $f=\nabla \cdot \nabla^{-2} \mathbf{u}$,

$$
(\mathbf{u} \cdot \mathbf{E}) \Psi_{J}(\xi)=\Psi_{J}(\xi)(\mathbf{e} \cdot \mathbf{E})+\frac{e}{\epsilon_{0}} \Psi_{J}\left(\nabla \cdot \nabla^{-2} \mathbf{u} \xi\right),
$$

and, using the fact that $\nabla^{-2}$ is an integral operator with integral kernel $1 /(4 \pi|\mathbf{x}-\mathbf{y}|)$, and working with unsmeared fields, we obtain

$$
\mathbf{E}(\mathbf{x}) \Psi_{J}(\mathbf{y})=\Psi_{J}(\mathbf{y})\left[\mathbf{E}(\mathbf{x})+\nabla \frac{e}{4 \pi \epsilon_{0}|\mathbf{x}-\mathbf{y}|}\right] .
$$

This can be interpreted as saying that creating a fermion using $\Psi(\mathbf{y})$ also creates its Coulomb field. Similar ideas appear, without the framework of induced algebras, in [13, Sections 79-80].

\section{Rieffel inducing}

Despite their advantage of being explicit, the above procedures do not easily extend to nonabelian gauge theories. (Apart from the obvious difficulty that the $\Omega$ action only preserves the inducing constraint in the abelian case, there can be obstructions to extensions in the nonabelian case $[10,11]$.)

In extending the approach it is useful to work with Rieffel's bimodule inducing [22], which like Mackey's construction, allows one to induce modules as well as algebras. Initially we shall just do this in an algebraic setting, ignoring the Hilbert structure. Let $\mathcal{B}$ and $\mathcal{C}$ be algebras, and $\mathcal{E}$ a $\mathcal{B}-\mathcal{C}$-bimodule, (that is, a left $\mathcal{B}$, right $\mathcal{C}$-module, with commuting actions). Then, from from a left $\mathcal{C}$-module $M$ one can induce a left $\mathcal{B}$-module $\mathcal{E} \otimes_{\mathcal{C}} M$, the quotient of $\mathcal{E} \otimes M$ by the subspace generated by

$$
\{e \cdot c \otimes m-e \otimes c \cdot m: e \in \mathcal{E}, c \in \mathcal{C}, m \in M\} .
$$

The $\mathcal{B}$-action is given by $b \cdot\left(e \otimes_{\mathcal{C}} m\right)=(b \cdot e) \otimes_{\mathcal{C}} m$. 
When $\mathcal{C}$ is a group algebra the quotient $\mathcal{E} \otimes_{\mathcal{C}} M$ can be expressed more simply, by regarding $\mathcal{E}$ and $M$ as having a group action. For $h$ in the group we require that $e \cdot h \otimes m=e \otimes h \cdot m$ in the quotient, or, equivalently, $e \otimes m=e \cdot h^{-1} \otimes h \cdot m$. This is just the requirement that we are in the fixed point subspace under the action $h: e \otimes m \mapsto e \cdot h^{-1} \otimes h \cdot m$.

Omitting the technical details, Rieffel's method extends this algebraic theory to $\mathrm{C}^{*}$-algebras $\mathcal{B}$ and $\mathcal{C}$ by assuming that the bimodule $\mathcal{E}$ has a $\mathcal{C}$-valued inner product, $\mathcal{E} \times \mathcal{E} \rightarrow \mathcal{C}$, which is $\mathcal{C}$-linear in the second variable

$$
\left\langle e_{1}, e_{2} \cdot c\right\rangle=\left\langle e_{1}, e_{2}\right\rangle \cdot c
$$

and $*_{\text {-symmetric }}$

$$
\left\langle e_{1}, e_{2}\right\rangle^{*}=\left\langle e_{2}, e_{1}\right\rangle,
$$

as well as positive $\langle e, e\rangle \geq 0$, that is, positive in the $\mathrm{C}^{*}$-algebra $\mathcal{C}$. When the $\mathcal{C}$-module $M$ has a Hilbert space structure consistent with the $\mathrm{C}^{*}$-algebra structure of $\mathcal{C}$ we can endow $\mathcal{E} \otimes M$ with the inner product

$$
\left\langle e_{1} \otimes m_{1}, e_{2} \otimes m_{2}\right\rangle=\left\langle m_{1},\left\langle e_{1}, e_{2}\right\rangle \cdot m_{2}\right\rangle .
$$

In general this is not positive definite so, to get a Hilbert space, we need to factor out by the radical (the vectors orthogonal to everything). We now note that

$$
\left\langle e_{1}, e_{2} \cdot c\right\rangle \cdot m_{2}=\left\langle e_{1}, e_{2}\right\rangle \cdot\left(c \cdot m_{2}\right),
$$

so that $\left(e_{2} \cdot c\right) \otimes m_{2}-e_{2} \otimes\left(c \cdot m_{2}\right)$ is always in the radical. This means that the quotient by the radical will be a quotient of $\mathcal{E} \otimes_{\mathcal{C}} M$, showing the connection with the algebraic approach. (Under suitable assumptions the inner product is positive definite on $\mathcal{E} \otimes_{\mathcal{C}} M$, and we shall assume this to be the case.)

We shall take $\mathcal{C}$ to be a convolution algebra of functions on $\mathcal{G}$, and $\mathcal{B}$ to be the boson algebra consistent with our previous notation. It turns out that an appropriate bimodule is the algebra $\mathcal{E}$ for the boson fields when gauge symmetries are ignored. (For example, in the abelian case we would take

$$
\mathcal{E}=\operatorname{ind}_{\{1\}}^{\Omega}(\operatorname{Cliff}(W, Q), \alpha) \rtimes_{\tau} \Omega \cong C(\Omega, \operatorname{Cliff}(W, Q)) \rtimes_{\tau} \Omega,
$$

instead of $\operatorname{ind}_{\mathcal{G}}^{\Omega}(\operatorname{Cliff}(W, Q), \alpha) \rtimes_{\tau} \Omega$, but, in general, we could use any well-defined *-algebra $\mathcal{E}$ for the bosons ignoring gauge symmetries, along with a compatible spacetime description of the fermions.) We assume that there is a $\mathcal{G}$-action $\alpha$ on $\mathcal{E}$ as before, and then the generalized fixed point algebra (in the multiplier algebra of $\mathcal{E}$ ) is $\mathcal{B}=\mathcal{E}^{\mathcal{G}}$, which has a multiplication action on $\mathcal{E}$ commuting with the action of $\mathcal{G}$, so that we may use Rieffel inducing to induce $\mathcal{G}$-modules to modules for $\mathcal{B}$. (The amenability of $\mathcal{G}$ enables one to prove the existence of generalized fixed point subspaces, although that can also be done explicitly. See also [8].) The algebra $\mathcal{E}$ is a group algebra of a nilpotent group, a central extension of the vector group $\Omega \times \Omega$, and it is therefore 
amenable. A pre-inner product $\left\langle e_{1} \mid e_{2}\right\rangle$ on $\mathcal{E}$ can be constructed by taking the invariant mean of the product $e_{1}^{*} e_{2}$, and we obtain the $\mathcal{C}$-valued inner product by defining

$$
\left\langle e_{1}, e_{2}\right\rangle(\chi)=\left\langle e_{1}, \chi \cdot e_{2}\right\rangle,
$$

which turns out to have the correct properties, provided that we specify the gauge group algebra $\mathcal{C}$ to include these functions.

Since we have a group algebra, the inducing procedure takes a $\mathcal{G}$-module $M$ to $F(M)=\mathcal{E} \otimes_{\mathcal{G}} M=(\mathcal{E} \otimes M)^{\mathcal{G}}$, where the tensor product action of $\chi \in \mathcal{G}$ sends $\mu \otimes m$ to $\alpha_{\chi}[\mu] \otimes \chi \cdot m$. (Strictly speaking, we should induce from the group algebra, but this is equivalent and simpler.)

For any $\mathcal{G}$-intertwiner $f$ between $\mathcal{G}$-modules $M \rightarrow N$, we can define

$$
F(f): \mu \otimes m \mapsto \mu \otimes f(m),
$$

which commutes with the $\mathcal{G}$ action, and so preserves the gauge fixed algebra. The action of $F(f)$ on the second tensor factor commutes with the actions of $\mathcal{B}$ on the first, so that $F(f)$ is a $\mathcal{B}$-morphism and $F$ defines a functor.

EXAMPLE 1. Consider the case of $M=\mathbb{C}$ the trivial $\mathcal{G}$ module. The induced module can be determined by using the generator $1 \in \mathbb{C}$, which enables one to identify $\mathcal{E} \otimes \mathbb{C}$ with $\mathcal{E}$ by $\mu \otimes 1 \mapsto \mu$. Under this identification $(\mathcal{E} \otimes \mathbb{C})^{\mathcal{G}}=\mathcal{E}^{\mathcal{G}}$, so that $F(\mathbb{C})$ is the fixed point algebra $\mathcal{E}^{\mathcal{G}}=\mathcal{B}$. The algebra product is inherited from that on $\mathcal{E}$ and the normal product on $\mathbb{C}$.

TheOREM 2. The map $F: M \mapsto \mathcal{E} \otimes_{\mathcal{G}} M$ from $\mathcal{G}$-modules to $\mathcal{B}$-bimodules, which takes a $\mathcal{G}$-intertwining operator $f: M \rightarrow N$ to $F(f): \mu \otimes_{\mathcal{G}} m \mapsto \mu \otimes_{\mathcal{G}} f(m)$, defines a functor from $\mathcal{G}$-modules and intertwiners to $\mathcal{B}$-bimodules and intertwiners.

PROOF. We have proved most of this except the statements about $\mathcal{B}$-bimodules. Since $\mathcal{G}$ acts by automorphisms of $\mathcal{E}$, we have, denoting the product of $\mu, \nu \in \mathcal{E}$ by $\mu * \nu$, $\alpha_{\chi}[\mu * \nu]=\alpha_{\chi}[\mu] * \alpha_{\chi}[\nu]$. When $\mu \in \mathcal{E}^{\mathcal{G}}$ this gives

$$
\alpha_{\chi}[\mu * v] \otimes \chi \cdot n=\mu * \alpha_{\chi}[\nu] \otimes \chi \cdot n=(\mu \otimes 1) * \chi(\nu \otimes n),
$$

so that we can define an action of $\mathcal{B}=\mathcal{E}^{\mathcal{G}}$ on $(\mathcal{E} \otimes N)^{\mathcal{G}}$ by $\mu *[\nu \otimes n]=(\mu * v) \otimes n$. We could equally well have used right multiplication by $f$, so that $F(M)$ is a $\mathcal{B}$ bimodule action. The action commutes with the $\mathcal{G}$ intertwining operators, which affect the other factor in the tensor product.

\section{A monoidal functor}

A strict monoidal or tensor category is a category $\mathcal{C}$, together with (i) an associative bifunctor $\otimes: \mathcal{C} \times \mathcal{C} \rightarrow \mathcal{C}$, and (ii) a unit object $U$ such that $U \otimes A \cong A \cong A \otimes U$ for all objects $A$, satisfying the obvious consistency conditions that the isomorphisms agree for $U \otimes U \cong U$, and for

$$
A \otimes B \cong(A \otimes U) \otimes B \cong A \otimes(U \otimes B) \cong A \otimes B .
$$


EXAMPLE 3. The $\mathcal{G}$-modules form a monoidal category under the tensor product (with the tensor product action, $\chi \otimes \chi$, of $\mathcal{G}$ ), and with unit the trivial module $\mathbb{C}$. $\mathcal{B}$-bimodules form a monoidal category with tensor product $\otimes_{\mathcal{B}}$ and unit object $\mathcal{B}$.

The question now arises as to whether we can interpret $F: M \mapsto(\mathcal{E} \otimes M)^{\mathcal{G}}$ as a monoidal functor. If so then the unit object should be $F(\mathbb{C}) \cong \mathcal{B}$, suggesting that $F$ maps from the monoidal category of $\mathcal{G}$-modules to the monoidal category of $\mathcal{B}$-bimodules. The main extra piece of information needed is a map $F_{M N}$, for each pair of $\mathcal{G}$-modules $M$ and $N$, which takes $F(M) \otimes_{\mathcal{B}} F(N)$ to $F(M \otimes N)$. The obvious map is to start with

$$
\left(\mu \otimes_{\mathcal{G}} m\right) \otimes\left(v \otimes_{\mathcal{G}} n\right) \mapsto(\mu * v) \otimes_{\mathcal{G}}(m \otimes n)
$$

For $\beta \in \mathcal{B},\left((\mu * \beta) \otimes_{\mathcal{G}} m\right) \otimes\left(v \otimes_{\mathcal{G}} n\right)-\left(\mu \otimes_{\mathcal{G}} m\right) \otimes\left(\beta * v \otimes_{\mathcal{G}} n\right)$ maps to

$$
[((\mu * \beta) * v)-(\mu *(\beta * v))] \otimes_{\mathcal{G}}(m \otimes n)=0,
$$

so that the right-hand side depends only on $\left(\mu \otimes_{\mathcal{G}} m\right) \otimes_{\mathcal{B}}\left(v \otimes_{\mathcal{G}} n\right)$, and we can regard $F_{M N}$ as a morphism $F(M) \otimes_{\mathcal{B}} F(N)$ to $F(M \otimes N)$.

THEOREM 4. The functor $F$, together with

$$
F_{M N}:\left(\mu \otimes_{\mathcal{G}} m\right) \otimes_{\mathcal{B}}\left(\nu \otimes_{\mathcal{G}} n\right) \mapsto(\mu * v) \otimes_{\mathcal{G}}(m \otimes n)
$$

and the identification $\mathcal{B} \rightarrow F(\mathbb{C})$, already used, is a monoidal functor, from $\mathcal{G}$-modules with the normal tensor product to $\mathcal{B}$-bimodules with the tensor product $\otimes_{\mathcal{B}}$.

PROOF. Due to the associativity of the convolution multiplication we see that this is consistent with strict associativity $\xi \otimes(\eta \otimes \zeta) \rightarrow(\xi \otimes \eta) \otimes \zeta$. We have checked that the left-hand side makes sense, but we also have

$$
\begin{aligned}
\chi \cdot[(\mu \otimes m)] \otimes \chi \cdot[(\nu \otimes n)] & =\left(\left(\alpha_{\chi}[\mu] \otimes \chi \cdot m\right) \otimes\left(\alpha_{\chi}[\nu] \otimes \chi \cdot n\right)\right) \\
& \mapsto\left(\alpha_{\chi}[\mu * \nu] \otimes \chi \cdot(m \otimes n)\right) \\
& =\chi \cdot[(\mu * \nu) \otimes(m \otimes n)]
\end{aligned}
$$

so that products map $\mathcal{G}$-fixed elements to $\mathcal{G}$-fixed elements.

In the abelian case, we see that

$$
\begin{aligned}
\left(\phi_{a, u} \otimes m\right) \otimes\left(\phi_{b, v} \otimes n\right) & \mapsto\left(\phi_{a, u} * \phi_{b, v}\right) \otimes(m \otimes n) \\
& =e^{i b(u)} \phi_{a+b, u+v} \otimes(m \otimes n),
\end{aligned}
$$

so that $F_{M N}$ sets up an isomorphism. This means that we actually have a strong monoidal functor.

The noncommutativity of $\mathcal{E}$ leads to a braiding in the image category. 
THEOREM 5. When $F$ is a strong monoidal functor, the tensor category of $\mathcal{B}$-bimodules with tensor product $\otimes_{\mathcal{B}}$ is a braided category with symmetric braiding

$$
\Phi_{F}=F_{N M}^{-1} \circ F(\Phi) \circ F_{M N}: F(M) \otimes_{\mathcal{B}} F(N) \rightarrow F(N) \otimes_{\mathcal{B}} F(M),
$$

as in the diagram

$$
F(M) \otimes_{\mathcal{B}} F(N) \rightarrow F(M \otimes N) \rightarrow F(N \otimes M) \cong F(N) \otimes_{\mathcal{B}} F(M),
$$

where the outer maps are given by the consistency maps $F_{M N}$ and $F_{N M}^{-1}$ and the middle map is $F(\Phi)$.

PROOF. The tensor product of $\mathcal{G}$-modules is braided trivially by the flip $\mathcal{G}$-morphism $\Phi: m \otimes n \mapsto n \otimes m$. Thus

$$
F(M) \otimes_{\mathcal{B}} F(N) \rightarrow F(M \otimes N) \rightarrow F(N \otimes M) \cong F(N) \otimes_{\mathcal{B}} F(M),
$$

where the outer maps are given by the consistency maps $F_{M N}$ and $F_{N M}^{-1}$ and the middle map is $F(\Phi)$. Although this braiding is nontrivial it is symmetric, since $\Phi^{2}=$ id gives $F(\Phi)^{2}=$ id. For many purposes this is almost as good as being the standard flip braiding.

We can see that the braiding is nontrivial in the abelian case by an explicit calculation:

$$
\begin{aligned}
\left(\phi_{a, u} \otimes m\right) \otimes\left(\phi_{b, v} \otimes n\right) & \mapsto e^{i b(u)} \phi_{a+b, u+v} \otimes(m \otimes n) \\
\left(\phi_{b, v} \otimes n\right) \otimes\left(\phi_{a, e} \otimes m\right) & \mapsto e^{i a(v)} \phi_{a+b, u+v} \otimes(n \otimes m) .
\end{aligned}
$$

We can also easily check the symmetry in this case.

\section{Transferring fermionic structure to the whole QED algebra}

Using the functor $F$, any structures which can be defined categorically for $\mathcal{G}$-modules can now be defined for $\mathcal{B}$-modules.

The argument which gave the braiding similarly leads to the following result.

LEMMA 6. For any morphism of $\mathcal{G}$-modules $\phi: M \otimes N \rightarrow P$ there is a morphism of $\mathcal{B}$-bimodules $\phi_{F}: F(M) \otimes_{\mathcal{B}} F(N) \rightarrow F(P)$, defined by $\phi_{F}=F(\phi) \circ F_{M N}$.

This has many useful corollaries, such as the following.

COROLlaRY 7. The gauge group $\mathcal{G}$ acts as automorphisms of an algebra $\mathcal{A}$ if and only if its multiplication $\mu$ is a $\mathcal{G}$-morphism. In this case $F(\mathcal{A})$ is an algebra with multiplication $F(\mu) \circ F_{\mathcal{A A}}$.

PROOF. The multiplication map $\mu: \mathcal{A} \otimes \mathcal{A} \rightarrow \mathcal{A}$ admits $\mathcal{G}$ as algebra automorphisms if and only if $\mu$ intertwines the actions of $\mathcal{G}$ on $\mathcal{A} \otimes \mathcal{A}$ and $\mathcal{A}$, so that $\mu \circ(\chi \otimes \chi)=$ $\chi \circ \mu$, which is precisely the condition that $\mu$ be a morphism in the category. Under this condition, take $M=N=P=\mathcal{A}$. 
There is a similar argument for modules.

COROLlary 8. Let $\mathcal{A}$ be an algebra on which $\mathcal{G}$ acts as automorphisms, and $N$ a covariant $(\mathcal{A}, \mathcal{G})$-module defined by an action $a: \mathcal{A} \otimes N \rightarrow N$, on which $\mathcal{G}$ also acts in a covariant way, that is, $\chi A \chi^{-1}=\chi[A]$ for all $A \in \mathcal{A}$. Then $F(N)$ is an $F(\mathcal{A})$ module.

Proof. Take $M=\mathcal{A}$ and $P=N$.

COROLlary 9. Let $W$ be a $\mathcal{G}$-module equipped with a $\mathcal{G}$-invariant quadratic form $Q: W \otimes W \rightarrow \mathbb{C}$. Then $Q_{F}: F(W) \otimes_{\mathcal{B}} F(W) \rightarrow F(\mathbb{C})=\mathcal{B}$ is a $\mathcal{B}$-valued quadratic form on $F(W)$.

Proof. Take $M=N=W$ and $P=\mathbb{C}$.

In general, the functor $F$ takes any structure which can be defined in the category of $\mathcal{G}$ modules, to a similar structure in the new monoidal category.

The Clifford algebra $\operatorname{Cliff}(W, Q)$ can be defined as the universal unital complex algebra for maps $f$ from $W$ to an algebra $\mathcal{C}$ such that one has a commutative diagram

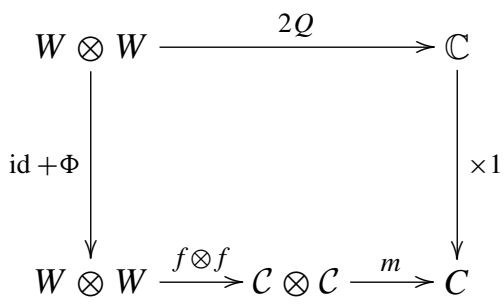

and for any such algebra $f$ there is a morphism $f_{*}: \operatorname{Cliff}(W, Q) \rightarrow \mathcal{C}$ whose composition with $W \rightarrow \operatorname{Cliff}(W, Q)$ is $f$.

THEOREM 10. The algebra $F(\operatorname{Cliff}(W, Q))$ is a universal object for the corresponding diagrams in the braided category of $\mathcal{B}$-bimodules, and so can be regarded as a Clifford algebra $\operatorname{Cliff}(F(W), F(Q))$ in that category.

This means that the algebra for interacting quantum electrodynamics can be regarded as a Clifford algebra over the gauge boson algebra, and inherits interesting features, coming from the trace, and antilinear anti-automorphism [21].

COROLlary 11. The fermion Clifford algebra has a unique braided commutative normalized conditional expectation $F(\tau): \operatorname{Cliff}(F(W), F(Q)) \rightarrow \mathcal{B}$, which is $\mathcal{B}$-linear.

Proof. There is a unique, hence $\mathcal{G}$-invariant, normalized trace $\tau: \operatorname{Cliff}(W, Q) \rightarrow \mathbb{C}$, and this gives a map $F(\tau): \operatorname{Cliff}(F(W), F(Q)) \rightarrow \mathcal{B}$. Linearity of the original trace 
is expressed by the commutativity of the diagram

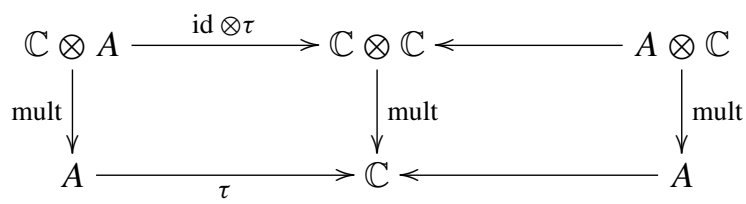

and application of $F$ shows that $F(\tau)$ is $\mathcal{B}$-linear (on both sides). Similarly, the trace property $\tau \circ$ mult $\circ \Phi=\tau \circ$ mult gives $F(\tau) \circ$ mult $\circ \Phi_{F}=F(\tau) \circ$ mult, showing that $F(\tau)$ is braided symmetric. Overall, $F(\tau)$ defines a braided symmetric conditional expectation from the full QED algebra to its bosonic part. Explicitly, we have $F(\tau)=\mathrm{id} \otimes \tau$.

This shows that, within the new category of $\mathcal{B}-\mathcal{B}$-bimodules, the type III QED algebra inherits some of the type $\mathrm{II}_{1}$ properties of the original Clifford algebra.

\section{Bar/monoidal dagger categories}

There is yet further structure in these categories. In order to be able to talk about antilinear operations such as a sesquilinear inner product on a Hilbert space or the $*$-structure on a $\mathrm{C}^{*}$-algebra it is useful to work in a bar or monoidal dagger category [1, 4, 27], which were devised for precisely this purpose. Bar categories are slightly more convenient for our purposes, as [4] already contains several examples of interest, so we shall use them with a change of notation.

A bar category has a functor from the category to its opposite, so that on objects bar : $A \mapsto \bar{A}$, with:

(i) a natural equivalence between the identity and bar obar functors; we shall actually assume a strong bar category, and identify $\overline{\bar{A}}=A$;

(ii) a natural morphism $U \mapsto \bar{U}$ from the unit object (which we shall just write as an identification);

(iii) a natural equivalence $\overline{(A \otimes B)} \rightarrow \bar{B} \otimes \bar{A}$ and consistency with the associator morphisms.

(We have abbreviated the conditions somewhat, the full definition is in [4].)

There is a natural functor on the category of $\mathcal{G}$-modules which takes a module $M$ to its conjugate $\bar{M}$ with the conjugate scalar multiplication by $\mathbb{C}$ and action of $\mathcal{G}$. A star object $M$ is one where there is an isomorphism $M \rightarrow \bar{M}$.

A *-algebra is a star object with the isomorphism $\mu \mapsto \mu^{*}$ from $M$ to $\bar{M}$. In particular, $\mathcal{E}$ and the fermionic Clifford algebra are star objects in the category of $\mathcal{G}$ modules. Moreover, there is also a bar structure on the $\mathcal{B}$-bimodules, [4, Example 2.3], and exploiting this with the braiding this means that we have isomorphisms

$$
\mathcal{E} \otimes \bar{M} \rightarrow \bar{M} \otimes \mathcal{E} \rightarrow \bar{M} \otimes \overline{\mathcal{E}} \rightarrow \overline{\mathcal{E} \otimes M},
$$


or $(\mu \otimes m)^{*}=\mu^{*} \otimes m^{*}$. From this it follows quickly that the fixed point sets agree:

$(\mathcal{E} \otimes \bar{M})^{\mathcal{G}} \cong \overline{(\mathcal{E} \otimes M)}$ G and $F(\bar{M}) \cong \overline{F(M)}$. (This can be seen by regarding the fixed points in $N$ as labelling the $\mathcal{G}$-morphisms $\mathbb{C} \rightarrow N$. Applying bar one has $\mathbb{C} \cong \overline{\mathbb{C}} \rightarrow \bar{N}$, which labels the fixed points of $\bar{N}$. Strictly we should have mapped into the multiplier algebra, but that is defined by a universal property for algebras having $N$ as a two-sided ideal.)

Putting all this together proves the following theorem.

THEOREM 12. The functor $F$ is a bar functor, that is, $F(\bar{M}) \cong \overline{F(M)}$.

Since $\tau\left[m^{*} m\right] \geq 0$ for the map $m \mapsto m^{*}$ on $\operatorname{Cliff}(W, Q)=\overline{\operatorname{Cliff}(W, Q)}$, and

$$
F(\tau)\left[(\mu \otimes m)^{*}(\mu \otimes m)\right]=\left(\mu^{*} \mu\right) \tau\left[m^{*} m\right],
$$

the trace $F(\tau)$ is positive, This means that we can form states $\phi \circ F(\tau)$ of $F$ (Cliff) by composing states $\phi$ of $\mathcal{B}$ with $F(\tau)$. Rieffel's imprimitivity theorem gives necessary and sufficient conditions for a given state to be of this form.

We note that whenever there is an inner product with values in the $*$-algebra $\mathcal{C}$, $\bar{M} \otimes M \rightarrow \mathcal{C}$, we have a map $\overline{F(M)} \otimes F(M) \rightarrow F(\mathcal{C})$. This gives an $F(\mathcal{C})$-valued inner product on $F(M)$, provided that it is positive.

\section{Carey's theorem}

The link between complex structures and fermionic vacuum states mentioned in Section 2 was clarified by Shale and Stinespring [28], who found necessary and sufficient conditions for two complex structures to define the equivalent representations of the Clifford algebra. An orthogonal transformation $T$ of the underlying inner product space $W$ gives rise to a Bogoliubov automorphism of the Clifford algebra, $\Psi(\xi) \mapsto \Psi(T \xi)$, and the Shale-Stinespring theorem also gave a criterion for this to be implemented by a unitary transformation of the representation space. In [7] Carey generalized this (and work of Blattner) to cover quasi-free representations of the Clifford algebra which are completely determined by their twopoint correlation functions. Robinson [23] recast the standard doubling construction used by Carey to show that every quasi-free representation of a Clifford algebra is obtained as a restriction of the regular representations on the Hilbert space

$$
\mathcal{H}_{\tau}=\left\{x \in \operatorname{Cliff}(W, Q)-\mathbb{C}: \tau\left(x^{*} x\right)<\infty\right\} .
$$

Writing $\lambda$ and $\rho$ for the left and right regular representations, and letting $\Gamma$ be the implementor of the orthogonal transformation -1 , we define

$$
\varpi(x \oplus y)=\lambda(x)+i \rho(y) \Gamma
$$

to obtain a Fock representation of $\operatorname{Cliff}(W \oplus W, Q \oplus Q)$ on $\mathcal{H}_{\tau}$ with vacuum vector the unit, 1. (Furthermore, the Tomita antiunitary operator coincides with the canonical conjugation on the Clifford algebra.) 
Robinson's construction used only the trace and *, from Clifford algebra theory, and those natural ingredients are now available for the generalized Clifford algebra Cliff $(F(W), F(Q))$ too. We hope to discuss their application to Carey's theorem in a subsequent paper.

\section{Conclusions}

The main conclusion of this paper is that algebra of fermions and gauge bosons can be regarded as a braided Clifford algebra $\operatorname{Cliff}\left(F(W), Q_{F}\right)$ over the braided commutative bosonic algebra $\mathcal{B}$, and that it shares many features with ordinary complex Clifford algebras. This enables one to incorporate the minimally coupled bosons as well as fermions, whilst retaining the spirit of the treatment of free fermions or those in classical external gauge fields, in, for example, [7, 11, 29, 31]. Nonetheless there are serious differences as soon as one studies the interacting fermion-boson dynamics. In a future paper we shall discuss applications of these ideas to interacting QED.

\section{References}

[1] S. Abramsky and B. Coecke, 'A categorical semantics of quantum protocols', Proceedings of the 19th IEEE Conference on Logic in Computer Science (LiCS'04) (IEEE Computer Society Press, Los Alamitos, CA, 2004).

[2] A. Ashtekar, Lectures on Non-perturbative Canonical Gravity (World Scientific, Singapore, 1991).

[3] A. Ashtekar and J. Lewandowsi, 'Representation theory of analytic holonomy C*-algebras', in: Knots and Quantum Gravity, Oxford Lecture Series in Mathematics and its Applications, 1 (ed. J. Baez) (Oxford University Press, New York, 1994).

[4] E. Beggs and S. Majid, 'Bar categories and star operations', Algebr. Represent. Theory 12 (2009), 103-152.

[5] P. Bouwknegt, K. C. Hannabuss and V. Mathai, 'Nonassociative tori and applications to T-duality', Comm. Math. Phys. 264 (2006), 41-69.

[6] A. L. Carey, 'Lorentz invariant wave equations', MSc dissertation, Oxford, 1973.

[7] A. L. Carey, 'Inner automorphisms of hyperfinite factors and Bogoliubov transformations', Ann. Inst. H. Poincaré 40 (1984), 141-149.

[8] A. L. Carey, J. M. Gaffney and C. A. Hurst, 'A $C^{*}$-algebra formulation of the quantization of the electromagnetic field', J. Math. Phys. 18 (1977), 629-640.

[9] A. L. Carey and H. Grundling, 'On the problem of the amenability of the gauge group', Lett. Math. Phys. 68 (2004), 113-120.

[10] A. L. Carey, H. Grundling, C. A. Hurst and E. Langmann, 'Realizing 3-cocycles as obstructions', J. Math. Phys. 36 (1995), 2605-2620.

[11] A. L. Carey, H. Grundling, I. Raeburn and C. Sutherland, 'Group actions on C*-algebras, 3-cocycles and quantum field theory', Comm. Math. Phys. 168 (1995), 389-416.

[12] A. Connes and D. Kreimer, 'Hopf algebras, renormalization and noncommutative geometry', Comm. Math. Phys. 199 (1998), 203-242.

[13] P. A. M. Dirac, Principles of Quantum Mechanics, 4th edn (Clarendon Press, Oxford, 1958).

[14] F. Dyson, 'The radiation theories of Tomonaga, Schwinger, and Feynman', Phys. Rev. 75 (1949), 486-502.

[15] H. Epstein and V. Glaser, 'The role of locality in perturbation theory', Ann. Inst. H. Poincaré Sect. A (N.S.) 19 (1973), 211-295. 
[16] K. Fredenhagen, K.-H. Rehren and E. Seiler, Quantum Field Theory: Where We Are. Approaches to Fundamental Physics, Lecture Notes in Physics, 721 (Springer, Berlin, 2007), pp. 61-87.

[17] J. M. Gracia-Bondia, J. C. Varilly and H. Figueroa, Elements of Noncommutative Geometry (Birkhäuser, Boston, 2001).

[18] H. Grundling and H. Neeb, 'Full regularity for a $\mathrm{C}^{*}$-algebra of the canonical commutation relations', Rev. Math. Phys. 21 (2009), 587-613.

[19] D. Kreimer, Knots and Feynman Diagrams, Cambridge Lecture Notes in Physics, 13 (Cambridge University Press, Cambridge, 2000).

[20] V. Mathai and J. Rosenberg, ' $T$-duality for torus bundles with $\mathrm{H}$-fluxes via noncommutative geometry', Comm. Math. Phys. 253 (2005), 705-721.

[21] R. Plymen and P. L. Robinson, Spinors in Hilbert Space, Cambridge Tracts in Mathematics, 114 (Cambridge University Press, Cambridge, 1994).

[22] M. A. Rieffel, 'Induced representations of C $C^{*}$-algebras', Adv. Math. 13 (1974), 176-257.

[23] P. L. Robinson, 'Modular theory and Bogoliubov autmomorphisms of Clifford algebras', J. Lond. Math. Soc. (2) 49 (1994), 463-476.

[24] G. Scharf, Finite Quantum Electrodynamics (Springer, Berlin, 1989).

[25] S. S. Schweber, QED and the Men Who Made it (Princeton University Press, Princeton, NJ, 1994).

[26] J. Schwinger (ed.), Selected Papers on Quantum Electrodynamics (Dover Publications, New York, 1958).

[27] P. Selinger, 'Dagger compact closed categories and completely positive maps', Proceedings of the 3rd International Workshop on Quantum Programming Languages, Chicago, June 30July 1, 2005.

[28] D. Shale and W. F. Stinespring, 'States of the Clifford algebra', Ann. of Math. (2) 80 (1964), 365-381.

[29] M. Spera and T. Wurzbacher, 'Determinants, Pfaffians and quasi-free representations of the CAR algebra', Rev. Math. Phys. 10 (1998), 705-721.

[30] H. Takai, 'On a duality for crossed products of $C^{*}$-algebras', J. Funct. Anal. 19 (1975), 25-39.

[31] T. Wurzbacher, 'Fermionic second quantization and the geometry of the restricted Grassmannian', in: Infinite-Dimensional Kaehler Manifolds, Birkhäuser Series on DMVSeminars, 31 (eds. A. Huckleberry and T. Wurzbacher) (Birkhäuser, Basel, 2001).

\section{KEITH C. HANNABUSS, Balliol College, Oxford OX1 3BJ, England e-mail: kch@balliol.ox.ac.uk}

